\title{
SECOND ORDER DIFFERENTIAL EQUATIONS WITH TRANSCENDENTAL COEFFICIENTS
}

\author{
JOHN ROSSI ${ }^{1}$
}

\begin{abstract}
Let $w_{1}$ and $w_{2}$ be two linearly independent solutions to $w^{\prime \prime}+$ $A w=0$, where $A$ is a transcendental entire function of order $\rho(A)<1$. We show that the exponent of convergence $\lambda(E)$ of the zeros of $E=w_{1} w_{2}$ is either infinite or satisfies $\rho(A)^{-1}+\lambda(E)^{-1} \leq 2$. For $\rho(A)=\frac{1}{2}$, this answers a question of Bank.
\end{abstract}

\section{Introduction. In this paper we prove}

THEOREM 1. Let $A$ be a transcendental entire function of order $\rho(A)<1$. If $w_{1}$ and $w_{2}$ are two linearly independent entire solutions to

$$
w^{\prime \prime}+A w=0,
$$

then the exponent of convergence $\lambda(E)$ of the sequence of zeros of $E=w_{1} w_{2}$ is either infinite or satisfies

$$
\rho(A)^{-1}+\lambda(E)^{-1} \leq 2 .
$$

Specifically,

$$
\rho(A) \leq \frac{1}{2} \Rightarrow \lambda(E)=+\infty .
$$

In particular, the implication (1.3) answers a question posed to us by Bank. In [2] he and Laine proved that $\rho(A)<\frac{1}{2}$ implies $\lambda(E)=+\infty$. Their method, which combines the $\cos \pi \rho$ theorem and Wiman-Valiron theory, does not seem to cover the case $\rho=\frac{1}{2}$.

To prove Theorem 1, we develop another method based on the Beurling-Tsuji estimate for harmonic measure [6, p. 116]. The idea of using this estimate was suggested to us by an unpublished manuscript of Edrei in which he attacks a similar problem. By applying a related technique, the spread theorem [1], he proves that (2.1) can never hold if both $A(z)$ and $E(z)$ have orders less than one and $A(z)$ satisfies a strong regularity condition.

In an earlier unpublished version of this paper, the author proved (1.3) using the Beurling-Tsuji inequality (together with a modification of some regularity theorems concerning functions extremal for the $\cos \pi \rho$ theorem). Using the same inequality, L. C. Shen [5] proved independently that $\rho(A)<1$ implies $\lambda(E) \geq 1$. Theorem 1 generalizes both results.

Received by the editors October 29, 1984 and, in revised form, May 24, 1985. Presented to the Society at the Summer Meeting, Laramie, Wyoming, August 15, 1985.

1980 Mathematics Subject Classification. Primary 30D35; Secondary 34A20.

${ }^{1}$ Part of this research was carried out during a visit to the University of Illinois-Urbana and was partially funded by NSF grant MCS 82-00497. 
Bank has pointed out to us that Theorem 1 has a curious

COROLLARY 1. There does not exist an entire function $E, \rho(E)<1$, such that the value of $E^{\prime}(z)$ at every zero of $E(z)$ is \pm 1 .

This result is contained in the work of Shen [5] and should be credited to him. It follows since by Lemma $\mathrm{C}$ in $[\mathbf{3}]$ such a function would have to be the product of two linearly independent solutions of $(1.1)$ where, by $(2.3), \rho(A)<1$. Theorem 1 then says $\lambda(E)>1$ which implies that $\rho(E)>1$, a contradiction. We note that for $\rho(E)=\frac{1}{2}, E(z)=2 \sqrt{z} \sin \sqrt{z}$ almost provides a counterexample to Corollary 1 except at zero, where $E^{\prime}(0)=2$.

It is an open question whether (1.3) holds provided $\rho(A)<1$ or whether $(1.2)$ is sharp. More generally it is not known whether (1.3) holds if $\rho(A)$ does not equal an integer. It certainly does not hold if $\rho(A)$ equals an integer or infinity, $[\mathbf{2}, \S 5 \mathrm{~b}]$.

We would like to thank A. Edrei and S. Bank for some valuable conversations regarding this problem. We would also like to thank the referee for his careful reading of two versions of this paper and for his helpful comments.

2. Preliminaries. Let $w_{1}$ and $w_{2}$ be linearly independent entire solutions to (1.1). Set $E=w_{1} w_{2}$. Then, as in [2, p. 354],

$$
4 A=\left(E^{\prime} / E\right)^{2}-2\left(E^{\prime \prime} / E\right)-c / E^{2},
$$

where $c$ is the (constant) Wronskian of $w_{1}$ and $w_{2}$. We assume from now on that the zeros of $E$ have finite exponent of convergence and show that (2.1) implies (1.2).

We assume familiarity with some basic Nevanlinna theory. Thus, by (2.1) and the lemma of the logarithmic derivative, we have

$$
m(r, 1 / E)=\frac{1}{2} m(r, A)+O(\log (r T(r, E))),
$$

where $r \rightarrow \infty$ outside a set $G$ of finite measure. Adding $N(r, 1 / E)$ to both sides of (2.2), and appealing to the first fundamental theorem of Nevanlinna theory and the fact that $A$ and $E$ are entire, gives

$$
T(r, E)=N(r, 1 / E)+\frac{1}{2} T(r, A)+O(\log (r T(r, E)))
$$

as $r \rightarrow \infty, r \notin G$. It follows that we need only prove

$$
\rho(A)^{-1}+\rho(E)^{-1} \leq 2,
$$

for this will imply (1.2). Indeed if $(2.4)$ holds, then $\rho(A)<\rho(E)$ since $\rho(A)<1$. Thus, $\lambda(E)=\rho(E)$ by $(2.3)$.

Since the zeros of $E$ have finite exponent of convergence and $A$ has finite order, it also follows from (2.3) that the order of $E, \rho(E)<\infty$. Thus we have

LEMMA 1. Given $\varepsilon>0$ there exists $C=C(\varepsilon)$ such that

$$
\left|\left(E^{\prime} / E\right)^{2}\left(r e^{i \theta}\right)-\left(2 E^{\prime \prime} / E\right)\left(r e^{i \theta}\right)\right| \leq r^{C}
$$

for all $r \geq r_{0}>1$ and all $\theta \notin J(r)$, where the angular measure of $J(r), m(J(r)) \leq$ $\varepsilon \pi$.

Proof. Let $H=\left(E^{\prime} / E\right)^{2}\left(r e^{i \theta}\right)-\left(2 E^{\prime \prime} / E\right)\left(r e^{i \theta}\right)$. Clearly

$$
m(r, H) \leq m\left(r, E^{\prime \prime} / E^{\prime}\right)+3 m\left(r, E^{\prime} / E\right)+O(1) .
$$


Thus, by the lemma of the logarithmic derivative and the fact that $E$ (and $E^{\prime}$ ) have finite order, there exists a constant $K$ such that $m(r, H) \leq K \log r$ for all $r \geq r_{0}$. Now fix $\varepsilon>0$ and let $C=2 K / \varepsilon$. Then by the definition of $m(r, H)$ and $K$ we easily have that for every $r \geq r_{0}$, the set $J(r)$ of $\theta$ where $\left|H\left(r e^{i \theta}\right)\right| \geq r^{C}$ has angular measure at most $\varepsilon \pi$. The proof is complete.

To state the next lemma we need some notation. Let $D$ be a region in $\mathbf{C}$. To each $r \in \mathbf{R}^{+}$set $\theta_{D}^{*}(r)=\theta^{*}(r)=+\infty$ if the entire circle $|z|=r$ lies in $D$. Otherwise, let $\theta_{D}^{*}(r)=\theta^{*}(r)$ be the measure of all $\theta$ in $[0,2 \pi)$ such that $r e^{i \theta} \in D$. As usual, the order $\rho(u)$ of a function $u$ subharmonic in the plane is given by $\rho(u)=\varlimsup_{\lim _{r \rightarrow \infty}} \log M(r, u) / \log r$, where $M(r, u)$ is the maximum modulus of $u$ on a circle of radius $r$.

LEMMA 2. Let $u$ be a subharmonic function in $\mathbf{C}$ and let $D$ be an open component of $\{z: u(z)>0\}$. Then

$$
\rho(u) \geq \varlimsup_{R \rightarrow \infty}(\log R)^{-1} \pi \int_{1}^{R} d t / t \theta_{D}^{*}(t) .
$$

Furthermore, given $\varepsilon>0$, define $F=\left\{r: \theta_{D}^{*}(r) \leq \varepsilon \pi\right\}$. Then

$$
\varlimsup_{R \rightarrow \infty}(\log R)^{-1} \int_{F \cap[1, R]} d t / t \leq \varepsilon \rho(u) .
$$

PROOF. An easy application of the maximum principle gives us $u$ unbounded in $D$. Thus we can find a point $z_{0} \in D$ such that $u\left(z_{0}\right) \geq 1$. By the Beurling-Tsuji inequality [6, p. 116] we obtain for $R \geq 4\left|z_{0}\right|$ that

$$
\log M(R, u) \geq \pi \int_{2\left|z_{0}\right|}^{R / 2} d t / t \theta_{D}^{*}(t)+C_{0},
$$

where $C_{0}$ is an absolute constant. Clearly (2.6) follows from (2.8). Also by (2.8) and the definition of $F$ we obtain

$$
\log M(R, u) \geq \varepsilon^{-1} \int_{F \cap\left[2\left|z_{0}\right|, R / 2\right]} d t / t+C_{0},
$$

from which (2.7) follows. The proof is complete.

We need one more lemma whose proof can be found in $[\mathbf{4}, \dot{6} 76]$. We prove it for completeness.

LEMMA 3. Let $l_{1}(t)>0, l_{2}(t)>0\left(t \geq t_{0}\right)$ be two measurable functions on $(0, \infty)$ with $l_{1}(t)+l_{2}(t) \leq(2+\varepsilon) \pi$, where $\varepsilon>0$. If $G \subseteq(0, \infty)$ is any measurable set and

$$
\pi \int_{G} d t / t l_{1}(t) \leq \alpha \int_{G} d t / t, \quad \alpha \geq 1 / 2
$$

then

$$
\pi \int_{G} d t / t l_{2}(t) \geq \frac{\alpha}{(2+\varepsilon) \alpha-1} \int_{G} d t / t .
$$

PROOF. By the Cauchy-Schwarz inequality

$$
\int_{G} d t / t l_{j}(t) \int_{G} l_{j}(t) d t / t \geq\left(\int_{G} d t / t\right)^{2}, \quad j=1,2 .
$$


From (2.10) and (2.12) with $j=1$ we obtain

$$
\int_{G} l_{1}(t) d t / t \geq \frac{\pi}{\alpha} \int_{G} d t / t .
$$

Thus

$$
\begin{aligned}
\int_{G} l_{2}(t) d t / t & \leq \int_{G}\left[(2+\varepsilon) \pi-l_{1}(t)\right] d t / t \\
& \leq\left[(2+\varepsilon) \pi-\frac{\pi}{\alpha}\right] \int_{G} d t / t .
\end{aligned}
$$

Substituting (2.14) into (2.12) with $j=2$ gives (2.11).

3. Proof of Theorem 1. Fix $\varepsilon>0$ and let $N$ be an integer such that

$$
N>C=C(\varepsilon)
$$

where $C$ is as in Lemma 1 and

$$
\log M(2, A)<N \log 2 .
$$

Since $A$ is transcendental there exists $z_{0},\left|z_{0}\right|>2$, such that $\log \left|A\left(z_{0}\right)\right|>N \log \left|z_{0}\right|$. Let $D_{1}$ be the component of the set

$$
\{z: \log |A(z)|-N \log |z|>0\}
$$

containing $z_{0}$. Clearly $D_{1}$ is open and since (3.2) holds, $\log |A(z)|-N \log |z|$ is subharmonic in $D_{1}$ and identically zero on $\partial D_{1}$. Thus, if we define

$$
u(z)= \begin{cases}\log |A(z)|-N \log |z|, & z \in D \\ 0, & z \in \mathbf{C} \backslash D,\end{cases}
$$

we have that $u(z)$ is subharmonic in $\mathbf{C}$ with

$$
\rho(u) \leq \rho(A) .
$$

Let $D_{2}$ be any component of $\{z: \log |E(z)|>0\}$ and let $D_{3}=\left\{r e^{i \theta}: \theta \in J_{r}\right\}$, where $J_{r}$ is as in Lemma 1 . (Note that the definitions of $D_{1}$ and $D_{3}$ depend on $\varepsilon$.) If for our given $\varepsilon,\left(D_{1} \cap D_{2}\right) \backslash D_{3}$ contains an unbounded sequence $r_{n} e^{i \theta_{n}}$ we obtain from the definitions of $D_{1}, D_{2}$ and $D_{3}$, Lemma 1 and (2.1) that

$$
r_{n}^{N}<\left|A\left(r_{n} e^{i \theta_{n}}\right)\right|<r_{n}^{C}+c, \quad n=1,2, \ldots,
$$

and this clearly contradicts (3.1) for $n$ large enough.

Thus for arbitrary fixed $\varepsilon>0$, we may assume that $\left(D_{1} \cap D_{2}\right) \backslash D_{3}$ is bounded. This implies that for $r \geq r_{1} \geq r_{0}$

$$
K_{r}=\left\{\theta: r e^{i \theta} \in D_{1} \cap D_{2}\right\} \subseteq J_{r},
$$

and thus by Lemma 1 the angular measure of $K_{r}$ satisfies

$$
m\left(K_{r}\right) \leq \varepsilon \pi .
$$

(We remark that the proof of Theorem 1 would now follow easily from (2.6) and Lemma 3 if $D_{1}$ and $D_{2}$ were disjoint. As we shall see, (3.4), (2.5) and (2.7) imply that these sets are "essentially" disjoint.) 
Define

$$
\begin{aligned}
& l_{1}(t)= \begin{cases}2 \pi & \text { if } \theta_{D_{1}}^{*}(t)=\infty, \\
\theta_{D_{1}}^{*}(t) & \text { otherwise, }\end{cases} \\
& l_{2}(t)= \begin{cases}2 \pi & \text { if } \theta_{D_{2}}^{*}(t)=\infty, \\
\theta_{D_{2}}^{*}(t) & \text { otherwise }\end{cases}
\end{aligned}
$$

Since $D_{1}$ and $D_{2}$ are unbounded open sets we have that $l_{1}(t)>0, l_{2}(t)>0$ for $t$ sufficiently large. Also, (3.4) gives $l_{1}(t)+l_{2}(t) \leq(2+\varepsilon) \pi$. Now let

$$
\varlimsup_{R \rightarrow \infty}(\log R)^{-1} \pi \int_{1}^{R} d t / t l_{1}(t)=\alpha .
$$

By definition of $l_{1}, \alpha \geq 1 / 2$. Since $l_{1}$ and $l_{2}$ satisfy the hypotheses of Lemma 3 , we obtain

$$
\varlimsup_{R \rightarrow \infty}(\log R)^{-1} \pi \int_{1}^{R} d t / t l_{2}(t) \geq \frac{\alpha}{(2+\varepsilon) \alpha-1} .
$$

Define $B_{j}=\left\{r: \theta_{D_{j}}^{*}(r)=\infty\right\}, j=1,2$. If $r \in B_{1}, r \geq r_{1}$, we have $\theta_{D_{2}}^{*}(r) \leq \varepsilon \pi$ by (3.4). Thus $B_{1} \subseteq\left\{r: \theta_{D_{2}}^{*}(r) \leq \varepsilon \pi\right\}$. By (2.7) we have

$$
\varlimsup_{R \rightarrow \infty}(\log R)^{-1} \int_{B_{1} \cap[1, R]} d t / t \leq \varepsilon \rho(E) .
$$

Let $\tilde{B}_{j}=\mathbf{R}^{+} \backslash B_{j}, j=1,2$. Then (3.3), (2.6) and (3.7) give

$$
\begin{aligned}
\rho(A) \geq \rho(u) & \geq \varlimsup_{R \rightarrow \infty}(\log R)^{-1} \pi \int_{1}^{R} d t / t \theta_{D_{1}}^{*}(t) \\
& =\varlimsup_{R \rightarrow \infty}(\log R)^{-1} \pi \int_{\tilde{B}_{1} \cap[1, R]} d t / t \theta_{D_{1}}^{*}(t) \\
& =\varlimsup_{R \rightarrow \infty}(\log R)^{-1}\left[\pi \int_{1}^{R} d t / t l_{1}(t)-\frac{1}{2} \int_{B_{1} \cap[1, R]} d t / t\right] \\
& \geq \alpha-(\varepsilon / 2) \rho(E) .
\end{aligned}
$$

Similarly,

$$
\varlimsup_{R \rightarrow \infty}(\log R)^{-1} \int_{B_{2} \cap[1, R]} d t / t \leq \varepsilon \rho(u)
$$

and

$$
\rho(E) \geq \varlimsup_{R \rightarrow \infty}(\log R)^{-1}\left[\pi \int_{1}^{R} d t / t l_{2}(t)-\frac{1}{2} \int_{B_{2} \cap[1, R]} d t / t\right] .
$$

Thus by (3.9), (3.10) and (3.6) we obtain

$$
\rho(E) \geq \frac{\alpha}{(2+\varepsilon) \alpha-1}-(\varepsilon / 2) \rho(u) .
$$

Inequalities (3.8) and (3.11) give

$$
\rho(E) \geq \frac{\rho(A)+(\varepsilon / 2) \rho(E)}{(2+\varepsilon)(\rho(A)+(\varepsilon / 2) \rho(E))-1}-(\varepsilon / 2) \rho(u) .
$$

Since $\varepsilon$ was arbitrary we obtain

$$
\rho(E) \geq \frac{\rho(A)}{2 \rho(A)-1} .
$$

This proves (2.4) and hence (1.2). The proof of Theorem 1 is complete. 


\section{REFERENCES}

1. A Baernstein II, Proof of Edrei's spread conjecture, Proc. London Math. Soc. (3) 26 (1973), 418-434.

2. S. Bank and I. Laine, On the oscillation theory of $f^{\prime \prime}+A f=0$ where $A$ is entire, Trans. Amer. Math. Soc. 273 (1982), 351-363.

3. __ On the zeros of meromorphic solutions of second order linear differential equations, Comment. Math. Helv. 58 (1983), 656-677.

4. A. Eremenko, Growth of entire and meromorphic functions on asymptotic curves, Sibirsk Mat. Zh. 21 (1980), 39-51; English transl. in Siberian Math. J. (1981), 673-683.

5. L. C. Shen, On a problem of Bank and Laine concerning the product of two linear independent solutions to $y^{\prime \prime}+A y=0$ (to appear).

6. M. Tsuji, Potential theory in modern function theory, Maruzen, Tokyo, 1959.

Department of Mathematics, Virginia Polytechnic Institute and State UNIVERSITY, BLACKSBURG, VIRGINIA 24061 